\title{
FORMULATION, DEVELOPMENT, AND CHARACTERISATION OF CILNIDIPINE LOADED SOLID LIPID NANOPARTICLES
}

\author{
REMYA PN, DAMODHARAN N*
}

Department of Pharmaceutics, SRM College of Pharmacy, SRM Institute of Science and Technology, Kanchipuram, Tamil Nadu, India. Email: damodharan.n@ktr.srmuniv.ac.in

Received: 08 January 2018, Revised and Accepted: 14 May 2018

\section{ABSTRACT}

Objective: The aim of the present investigation is to develop solid lipid nanoparticles (SLNs) of cilnidipine using hot homogenization followed by ultrasonication technique and to improve the dissolution characteristics of the drug.

Methods: The cilnidipine-loaded SLNs were formulated using stearic acid (SA), glyceryl monostearate (GMS), and palmitic acid (PA) as lipid matrix and tween-20, tween-80, and tween-40 as an emulsifier by hot homogenization and ultrasonication method. The physicochemical characteristics of SLN were analyzed for Fourier transform infrared studies, entrapment efficiency (EE), zeta potential, in vitro drug release, particle size analysis, scanning electron microscopy, and stability.

Results: The SLNs with PA showed a sustained release of drug 82\%-88\%, respectively, after 10 h. The SLNs of PA using tween-80 as emulsifier resulted with high EE\% than SLNs of SA and GMS. The compatibility studies are done by Fourier transformed infrared for formulations which contain PA as lipid matrix and tween-80 as an emulsifier, and it showed no drug excipient incompatibility. The formulation containing PA and tween-80 shown particles of average size $152 \mathrm{~nm}$ having polydispersity index of. 217 with $68.7 \%$ EE were produced. The zeta potential of the formulation was found to be $-27 \mathrm{mV}$ and the order of percentage drug release was from $\mathrm{PA}>\mathrm{GMS}>\mathrm{SA}$, and steric stabilizers retard the drug release more than ionic stabilizers.

Conclusion: SLN formulations showed the best results in EE as well as in in vitro drug release and therefore confirmed that the novel drug delivery system provides an improved strategy for the treatment of hypertension.

Keywords: Cilnidipine, Solid lipid nanoparticles, Stearic acid, Glyceryl monostearate, Palmitic acid, Tween-20, Tween-80, Tween-40.

(C) 2018 The Authors. Published by Innovare Academic Sciences Pvt Ltd. This is an open access article under the CC BY license (http://creativecommons. org/licenses/by/4. 0/) DOI: http://dx.doi.org/10.22159/ajpcr.2018.v11i9.24666

\section{INTRODUCTION}

Solid lipid nanoparticles (SLNs) are particles prepared from solid lipids, with a mean diameter in the range from 50 to $1000 \mathrm{~nm}$. It has become one of the important active areas of research in the field of drug delivery because of their ability to deliver drugs to the right place, at appropriate times, and in the right dosage [1-3]. They have achieved considerable attention over the past 20 years due to their advantages compared to other drug delivery systems. SLNs have increased attention in pharmaceutics as an alternative dosage form to liposomes and polymeric nanoparticles. SLNs shown good tolerability and stability, scaling-up feasibility, and the ability to incorporate hydrophobic/hydrophilic drugs [4]. The incorporation of poorly soluble drugs as SLN can improve gastrointestinal solubilization, absorption, and bioavailability of the drug [5-8].The present research showed that SLN could significantly extend systemic circulation times, increase the bioavailability, reduce the dose, frequency of administration, and decrease side effects $[9,10]$.

Cilnidipine, a novel dihydropyridine calcium channel blocker found reported to exhibit excellent clinical effects on cardiovascular diseases. A unique pharmacological property for cilnidipine is that it inhibits both L-type and N-type calcium channels in various types of neurons. Recently, cilnidipine was found to possess much more unique advantages compared with traditional calcium channel blockers, for example; it causes a lower probability of reflex tachycardia and less effect on heart rate as compared to nifedipine. In addition, the renal protective effects of cilnidipine imply its great potency in benefiting hypertensive patients in altering their blood pressures [11]. However, the dissolution and oral bioavailability of cilnidipine are not as good as expected, mainly because of its poorly water-soluble property. The aim of this study was to prepare and characterize the cilnidipine loaded SLN using stearic acid (SA), palmitic acid (PA), and glyceryl monostearate as lipid matrix and tween-20, tween-40, and tween-80 as emulsifier by a hot homogenization and ultrasonication method with a view to improving the dissolution rate of cilnidipine which could increase the biological activities [12].

\section{MATERIALS AND METHODS}

\section{Materials}

All the chemicals and reagents required for the present research work has been obtained from the authentic supplier and were of analytical grade. The API, cilnidipine has been obtained as the gift sample from Madras Pharmaceuticals, Chennai, India. SA, PA, glyceryl monostearate (GMS) was obtained from SIS-Lab Fine Chem. Industries, Chennai India. Tween-20, Tween-40, Tween-80, acetone, methanol, and hydrochloric acid were purchased from the local market. Triple distilled water obtained at SRM College of Pharmacy, SRM University, Chennai, was used throughout the experiment.

\section{Preparation of SLNs}

The cilnidipine SLNs were prepared by hot homogenization followed by the ultrasonication method. The different lipids were used in the concentration of $0.5-1.5 \%$ and the surfactant tween $(20,40$, and 80 ) was used in the concentration of $1 \%, 1.5 \%$, and $2 \%$. The drug, lipid, and soya lecithin were added together to get the organic phase of preparation. The lipid content in the organic phase was melted by heating at $70^{\circ} \mathrm{C}$, and the aqueous phase was prepared by dissolving hydrophilic surfactant tween ( 1 to $2 \% \mathrm{w} / \mathrm{w}$ ) by heating to the same temperature as the organic phase. The hot aqueous phase was added to the organic phase under magnetic stirring (Remi, Mumbai, 
India) at $1000 \mathrm{rpm}$ to form pre-emulsion. The hot pre-emulsion was then homogenized at 10,000 rpm for 3 min using the high-pressure homogenizer kept in a water bath maintained at $70^{\circ} \mathrm{C}$ then immediately placed in ultrasonicator at $75 \%$ amplitude for $25 \mathrm{~min}$. [13]. Different SLNs were prepared with the same concentration of soya lecithin, and all formulations are prepared with different concentrations of SA, PA, and GMS as shown in Tables 1-3 and abbreviated as A1-A9, B1-B9, F1F9, and so on.

\section{Fourier transformed infrared (FTIR) spectroscopic analysis}

An FTIR spectroscopy study was conducted to check the compatibility between the drug (Cilnidipine) and lipid (SA, PA, and GMS) separately which is used for the preparation of nanoparticles [14]. The FTIR was determined for drug, lipids and physical mixture of drug and lipid at a wavelength from 4000 to $400 \mathrm{~cm}^{-1}$.

\section{Particle size and zeta potential determination}

The average particle size, polydispersity index, and zeta potential of the lipid particulate dispersions were performed using a zeta sizer (DTS Ver.5.10, Malvern Instruments). The dispersion was diluted to $1: 10 \mathrm{v} / \mathrm{v}$ with double distilled water (filtered through $0.45 \mu \mathrm{m}$ membrane filters) to determine that the light scattering intensity was within the instrument's sensitivity range $[15,16]$.

\section{Determination of entrapment efficiency (EE)}

The EE was analyzed for the free drug content in the supernatant obtained after centrifuging the SLN suspension in high-speed centrifuge at $15000 \mathrm{rpm}$ for $30 \mathrm{~min}$ using Remi centrifuge (Mumbai, India). The EE was calculated as follows:

\section{$\mathrm{EE}=($ Total drug content - free drug content/Total drug content $) \times 100$}

\section{In vitro drug release}

In vitro release studies were undergone using dialysis bag diffusion technique. The dialysis membrane having pore size $2.4 \mathrm{~nm}$, molecular weight cutoff 12,000-14,000 was used. The membrane was soaked in double-distilled water for $12 \mathrm{~h}$ before loading the SLN dispersions containing the drug. An accurately weighed amount of cilnidipine loaded SLN dispersions containing the drug equivalent to $5 \mathrm{mg}$ was transferred to a dialysis bag and sealed. The sealed bag was then suspended in a beaker containing dialysis medium containing $250 \mathrm{ml}$ of phosphate buffer pH 7.4 and stirred at a constant speed of $50 \mathrm{rpm}$. An aliquot of $1 \mathrm{ml}$ of sample was withdrawn at time intervals of $0.5,1$, $2,4,6,8$, and $12 \mathrm{~h}$. Fresh medium was replaced each time to maintain a constant volume. Samples were analyzed by ultraviolet spectroscopy.

\section{Scanning electron microscopy (SEM)}

The morphology of the SLN was examined by SEM (Hitachi, Japan). The samples were stained with $2 \%(\mathrm{w} / \mathrm{v})$ phosphotungstic acid for $30 \mathrm{~s}$ and placed on copper grids with films for viewing.

\section{Stability studies}

The cilnidipine loaded SLNs were stored at room temperature $\left(25^{\circ} \mathrm{C}\right)$ and refrigerator temperature $\left(2-8^{\circ} \mathrm{C}\right)$ for 1 month and average particle size, zeta potential, and polydispersity index (PDI) were determined. The SLNs were stored at room temperature for 30 days, and EE is calculated.

\section{RESULTS}

In this study cilnidipine loaded SLNs were prepared by hot homogenization followed by ultrasonication method using different lipids and surfactants at different concentration and the results were tabulated in Tables 4 and 5. The mean particle size was found within a range of $131.32 \pm 39.93-339.67 \pm 30.95 \mathrm{~nm}$ (Fig. 4). Depending on the comparison of visual clarity and particle size, the formulation contains tween 80 and PA (F1, F4, and F7) having the mean particle size from $131.32 \pm 39.93$ to $187.68 \pm 37.92 \mathrm{~nm}$. The formulations were evaluated for PDI, zeta potential, and EE and are presented in Tables 4 and 5. From these studies, most efficient formulations of F7 with the particle size,
PDI, zeta potential, and EE were found to be $131.32 \pm 39.93 \mathrm{~nm}, 0.217$, $-28.9 \pm 3.052 \mathrm{mV}$, and $71.73 \pm 4.57 \%$, respectively, and considered as best preparation.

\section{Particle sizes and zeta potentials}

All the prepared formulations were determined for their particle size distributions and zeta potential values. The results obtained after particle size analysis are shown in Table 4 . All the formulations the particle sizes ranged from $131.32 \pm 39.93$ to $339.67 \pm 30.95 \mathrm{~nm}$ and zeta potential $-24.0 \pm 3.509$ to $-29.4 \pm 8.890 \mathrm{mV}$, and PDI was found to be between $0.202 \pm 0.057$ and $0.465 \pm 0.053$ (Table 4) indicating the narrow size distribution.

\section{EE and in vitro drug release}

About $78.73 \%$ of EE is seen in the F7 formulation, and it is due to the low content of lipid in formulation showed more drug release. The zeta potential is known to be predictive of the stability of nanoparticles $[17,18]$. The zeta potential of SLNs obtained was $-24.0 \pm 3.509-29.4 \pm 8.890 \mathrm{mV}$, which is considered to be highly stable. The EE and loading capacity of SLN were enhanced with increasing the carbon chain length of fatty acid since the higher hydrophobicity of the longer chain fatty acids resulted in increased accommodation of lipophilic drugs [19]. The longer carbon chain length of the fatty acid SLN had slower release rates in vitro; this was attributed to that the enhanced lipophilicity of longer chain fatty acids had better drug retaining capacity [20]. The in vitro drug release profile of SLN formulation showed a biphasic pattern with the initial rapid release of drug followed by a sustained release [21]. This is due to the presence of the drug in the outer region of SLN and F7 showed a maximum drug release of $89.42 \%$ during $12 \mathrm{~h}$ (Fig. 1).

\section{FTIR study}

The frequencies of functional groups of the drug cilnidipine remained same and showed the presence of an intact peak in a physical mixture as it is evident in $1697 \mathrm{~cm}^{-1}$ as the pure drug. Hence, it was concluded that there was no major interaction occurred between the drug and excipients used in the study.

\section{Stability study}

All the optimized formulations are stored in bottles at room temperature and refrigerator temperature. As SLN formulation A1, A4, and A7 are not stable and so rest of formulation are analyzed for particle size, zeta potential, and PDI. There is no significant difference in particle size, zeta potential and PDI of SLN formulation F1, F4, and F7 as the duration of storage increase up to 1 month the SLN formulation found to be stable. Due to the slow transition of lipid in nanoformulations and low particle size along with steric effect of Tween-80 enhances the stability of the proposed formulation [22].

\section{DISCUSSION}

\section{Formulation optimization}

Hot homogenization followed by ultrasonication was mentioned to be an economic, simple, reproducible and most good method for the preparation of SLNs. In this technique, the preparation of SLNs does not require any organic solvents, which could be difficult to remove after nanoparticle synthesis. In this method, the selection of lipid is crucial in the formulation design. Lipids are selected based on their ability to solubilize the drug. SA, PA, and GMS were selected because of its high dissolution of cilnidipine, even though other lipids such as hydrogenated castor oil had better characteristics used as lipid matrices to prepare SLN. Hydrogenated castor oil SLNs were an effective nanoparticle system for controlled release and enhancement of pharmacological activities of encapsulated drugs but cilnidipine hardly dissolved in the melted lipid. Cilnidipine had good retention in the drug-lipid mixture once it was dissolved. In the (Fig. 2) represents the IR Spectra of pure drug and the physical mixtures. It is absorbed that all major peaks of the drug obtained in the IR spectra shown no significant change in the IR spectra of drug excipients mixture (Fig. 4) The drug did not crystallize from the melted lipid even when the temperature of the mixture 
Table 1: Composition of various SLNs formulations with Tween-20

\begin{tabular}{llllllll}
\hline Formulation code & Cilnidipine (\%) & SA (\%) & GMS (\%) & PA (\%) & Soy lecithin (\%) & Tween-20 (\%) & Distilled water (ml) \\
\hline A1 & 0.1 & 0.5 & - & - & 0.50 & 2 & 100 \\
A2 & 0.1 & 1 & - & - & 0.50 & 1.5 & 100 \\
A3 & 0.1 & 1.5 & - & - & - & 2 & 100 \\
A4 & 0.1 & - & 0.5 & - & - & 1.5 & 100 \\
A5 & 0.1 & - & 1 & - & 0.50 & 1 & 100 \\
A6 & 0.1 & - & 1.5 & - & 0.50 & 2 & 100 \\
A7 & 0.1 & - & - & 0.5 & 0.50 & 1.5 & 100 \\
A8 & 0.1 & - & - & 1 & 0.50 & 1 & 100 \\
A9 & 0.1 & - & 1.5 & 0.50 & & \\
\hline
\end{tabular}

SLN: Solid lipid nanoparticles, SA: Stearic acid, GMS: Glyceryl monostearate, PA: Palmitic acid

Table 2: Composition of various SLNs formulations Tween-40

\begin{tabular}{llllllll}
\hline Formulation code & Cilnidipine (\%) & SA (\%) & GMS (\%) & PA (\%) & Soya lecithin (\%) & Tween-40 (\%) & Distilled water (ml) \\
\hline B1 & 0.1 & 0.5 & - & - & 0.50 & 2 & 1.5 \\
B2 & 0.1 & 1 & - & - & 0.50 & 1.5 & 100 \\
B3 & 0.1 & 1.5 & - & - & 0.50 & 2 & 100 \\
B4 & 0.1 & - & 0.5 & - & 0.50 & 1.5 & 100 \\
B5 & 0.1 & - & 1 & - & 0.50 & 1 & 100 \\
B6 & 0.1 & - & 1.5 & - & 0.50 & 2 & 100 \\
B7 & 0.1 & - & - & 0.5 & 0.50 & 1.5 & 100 \\
B8 & 0.1 & - & - & 1 & 0.50 & 1 & 100 \\
B9 & 0.1 & -1.5 & 0.50 & & \\
\hline
\end{tabular}

SLN: Solid lipid nanoparticles, SA: Stearic acid, GMS: Glyceryl monostearate, PA: Palmitic acid

Table 3: Composition of various SLNs formulations Tween-80

\begin{tabular}{llllllll}
\hline Formulation code & Cilnidipine (\%) & SA (\%) & GMS (\%) & PA (\%) & Soya lecithin (\%) & Tween-80 (\%) & Distilled water (ml) \\
\hline F1 & 0.1 & 0.5 & - & - & 0.50 & 2 & 100 \\
F2 & 0.1 & 1 & - & - & 0.50 & 10.5 & 100 \\
F3 & 0.1 & 1.5 & - & - & 0.50 & 2 & 100 \\
F4 & 0.1 & - & 0.5 & - & 0.50 & 1.5 & 100 \\
F5 & 0.1 & - & 1 & - & 0.50 & 1 & 100 \\
F6 & 0.1 & - & -1.5 & - & 0.50 & 1.5 & 100 \\
F7 & 0.1 & - & - & 0.5 & 0.50 & 1 & 100 \\
F8 & 0.1 & - & - & 1.5 & 0.50 & 1 & \\
F9 & 0.1 & - & - & 1.50 & & \\
\hline
\end{tabular}

SLN: Solid lipid nanoparticles, SA: Stearic acid, GMS: Glyceryl monostearate, PA: Palmitic acid

Table 4: Average particle size, zeta potential, and PDI of cilnidipine loaded SLN formulations

\begin{tabular}{llll}
\hline $\begin{array}{l}\text { Formulation } \\
\text { code }\end{array}$ & $\begin{array}{l}\text { Particle } \\
\text { size }(\mathbf{n m})\end{array}$ & PDI & $\begin{array}{l}\text { Zeta } \\
\text { potential } \mathbf{( m V})\end{array}$ \\
\hline A1 & $238.71 \pm 34.93$ & $0.262 \pm 0.052$ & $-29.6 \pm 5.050$ \\
A4 & $222.20 \pm 30.94$ & $0.244 \pm 0.072$ & $-28.4 \pm 7.560$ \\
A7 & $233.11 \pm 31.96$ & $0.269 \pm 0.068$ & $-29.4 \pm 8.890$ \\
B1 & $339.30 \pm 30.95$ & $0.465 \pm 0.053$ & $-27.6 \pm 7.775$ \\
B4 & $253.94 \pm 38.96$ & $0.271 \pm 0.065$ & $-24.2 \pm 3.056$ \\
B7 & $331.41 \pm 37.96$ & $0.290 \pm 0.037$ & $-28.5 \pm 2.095$ \\
F1 & $135.01 \pm 30.92$ & $0.202 \pm 0.057$ & $-29.0 \pm 3.457$ \\
F4 & $187.68 \pm 37.92$ & $0.234 \pm 0.074$ & $-24.0 \pm 3.509$ \\
F7 & $131.32 \pm 39.93$ & $0.217 \pm 0.066$ & $-28.9 \pm 3.052$ \\
\hline PDI: Polydispersity & & &
\end{tabular}

decreased from $150^{\circ} \mathrm{C}$ to $85^{\circ} \mathrm{C}$. The lipid concentration increased from 0.5 to $1.5 \%$, the mean particle sizes and particle size distribution also increased higher concentration of lipid content increased the viscosity of the lipid dispersion which affected the homogenization efficiency and increased rate of particle agglomeration; hence, the lipid content of the SLNs dispersion should not exceed 5\% [23]. Drug expulsion in SLN can occur when the lipid matrix transforms from high energy modifications characterized by the presence of many imperfections with forming a perfect crystal with no room for guest molecules. PA -lipid molecules are chemically different resulting in a structure with many imperfections to accommodate the drug and thus higher loading capacity. The right selection of surfactant with proper concentration was required to prepare physicochemically stable lipid nanoparticles $[24,25]$. In the present study, the SLNs dispersion stabilized by the surfactant ( $2 \%$ tween 80) had smaller particle size when compared with the formulation of other surfactants. From the optimization study, the suitable blank SLNs formulation containing $0.5 \%$ PA, $2 \%$ tween 80 , and $0.5 \%$ soya lecithin were considered for further incorporation of the drug.

\section{Formulation and characterization of cilnidipine SLNs}

The SLNs selected in the optimization study were used to entrap $0.1 \%$ cilnidipine using a hot homogenization coupled with ultrasonication method. A high energy such as high production temperature, high stirring rate, longer emulsification time, and stronger ultrasound power was applied in this study to get a finer dispersion of formulation. In the current study, the homogenization pressure 10,000 psi was applied for $3 \mathrm{~min}$ and followed by ultrasonication resulted in the mean \pm standard deviation (SD) particle size of $131.34 \pm 37.83 \mathrm{~nm}$ with narrow size distribution. The result suggests that the hot homogenization and ultrasonication method was a feasible and compatible method for preparing cilnidipine loaded PA SLNs.

The preparation resulted in consistent nanoparticles of narrow size distribution and resulted a slight increase in particle sizes from $131.32 \pm 39.93$ to $339.30 \pm 30.95 \mathrm{~nm}$. These findings are inconsistent with 


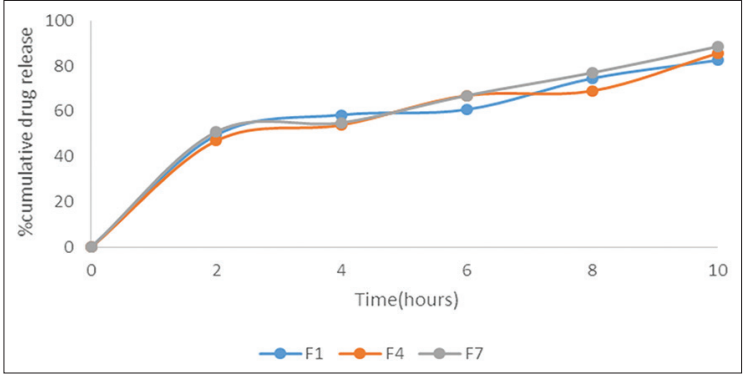

Fig. 1: In vitro drug release studies of selected batches

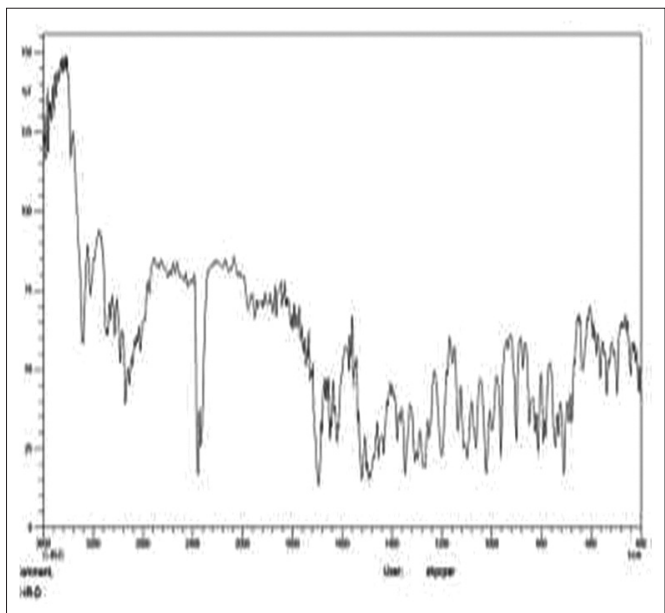

Fig. 2: Fourier transformed infrared spectra of cilnidipine

Table 5: EE and in vitro drug release of cilnidipine loaded SLN formulations

\begin{tabular}{llll}
\hline SI. No & $\begin{array}{l}\text { Formulation } \\
\text { code }\end{array}$ & EE (\%) & In vitro drug release (\%) \\
\hline 1 & A1 & $61.66 \pm 6.35$ & 84.92 \\
2 & A $~$ & $64.75 \pm 5.46$ & 83.91 \\
3 & A7 & $72.64 \pm 4.85$ & 84.23 \\
4 & B1 & $53.85 \pm 3.78$ & 82.03 \\
5 & B 4 & $54.22 \pm 7.75$ & 86.34 \\
6 & B7 & $61.35 \pm 5.67$ & 86.49 \\
7 & F1 & $66.73 \pm 8.49$ & 88.53 \\
8 & F4 & $67.80 \pm 6.33$ & 87.66 \\
9 & F7 & $71.73 \pm 4.57$ & 89.42 \\
\hline
\end{tabular}

EE: Entrapment efficiency, SLN: Solid lipid nanoparticles

the study of Jensen et al. [26] who explained that the increase in size of SLNs after incorporation of drug reflected the dissolution of the drug in the lipid phase. A narrow particle size distribution was an indication of nanoparticles stability and homogeneous dispersion [27]. PDI values ranging from 0 to 0.5 were considered to be monodisperse and homogenous, but those of more than 0.5 indicated non-homogeneity and polydispersity [26]. In the present study, the particle size distribution was monodisperse and homogenous as formulation has less mean \pm standard error PDI of $0.17 \pm 0$. According to Schwarz and Mehnert [27] and Zimmermann etal. [28], the negative charge of zeta potential was conferred by the lipids used in the SLNs. In agreement with this, the PA utilized in this study provided negative charge of zeta potential. Nanoparticle with zeta potential values $>+25 \mathrm{mV}$ or $<-25 \mathrm{mV}$ typically has high degrees of stability due to electric repulsion between particles. Dispersions with a low zeta potential value will aggregate due to Van Der Waal inter-particle attraction [21]. In this study, the mean $\pm S D$ zeta potential of $-14.2 \pm 1.00$

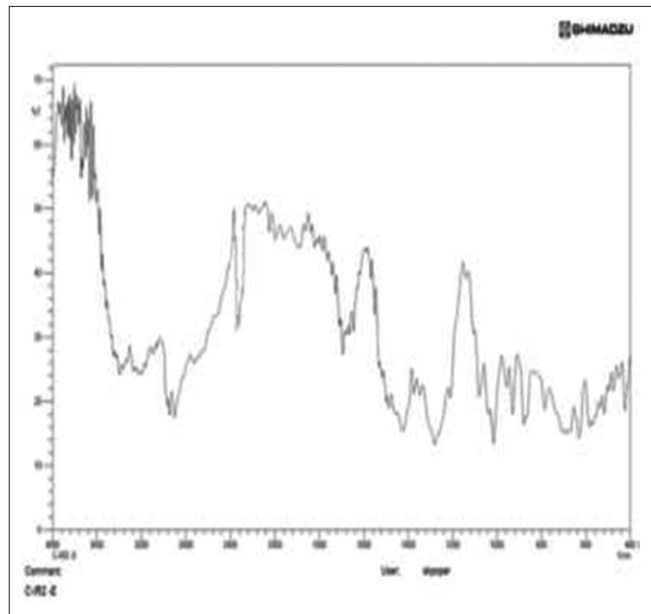

Fig. 3: Fourier transformed infrared spectra of cilnidipine and excipients

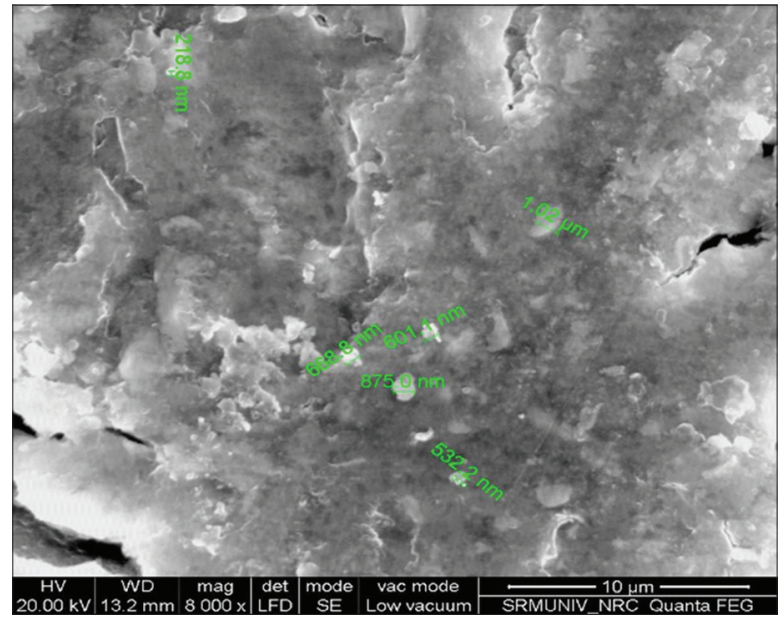

Fig. 4: Scanning electron microscopy image of optimized cilnidipine loaded solid lipid nanoparticles

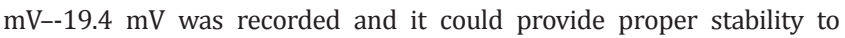
the cilnidipine SLNs. The small size of nanoparticles is favorable for improving the oral performance of incorporated poorly soluble drugs. The initial fast release of the drug could be due to desorption and diffusion of cilnidipine accumulated at the oil-water interface and in the outer shell of nanoparticles [29]. The initial release should be sufficiently rapid to ensure that the therapeutic drug levels are achieved in a timely manner in vivo. The subsequent slow release is mainly due to the slow diffusion of drug molecules through the lipid matrix of the nanoparticles $[30,31]$. Slow drug release will contribute to maintaining the effective therapeutic drug concentrations. The optimized cilnidipine SLNs were chosen as SLN F7 which shows a least zeta potential and good EE. As the concentration of surfactant mixture is increased showed smallest particle size and it has been found out that Tween 80 promotes the formation of smaller sized nanoparticles and were observed that increased content of surfactant promotes the formation of smaller nanoparticles [32]. All the SLNs formulations showed negative zeta potential values which indicate the stable nature of nanoparticles due to electrostatic repulsion. As the surfactant content increased, EE increases which could be due to the formation of stabilized nanoparticles [33-35].

\section{Stability studies}

Table 6 represents the results of stability studies for the prepared SLNs formulations. The drug EE and the physical appearance of the 
Table 6: Influence of storage condition and duration of storage on particle size, zeta potential, PDI, and EE

\begin{tabular}{|c|c|c|c|c|c|c|c|}
\hline Storage condition & $\begin{array}{l}\text { Formulation } \\
\text { code }\end{array}$ & Duration & $\begin{array}{l}\text { Particle } \\
\text { size }(\mathbf{n m})\end{array}$ & Zeta potential $(\mathrm{mV})$ & PDI & EE (\%) & $\begin{array}{l}\text { In vitro drug } \\
\text { release (\%) }\end{array}$ \\
\hline \multirow[t]{2}{*}{ Room temperature } & $\mathrm{F} 1$ & Day 1 & $235.3 \pm 39.2$ & $-29.0 \pm 3.45$ & $0.263 \pm 0.092$ & $56.73 \pm 4.45$ & 88.53 \\
\hline & & Day 30 & $238.7 \pm 30.92$ & $-29.4 \pm 2.96$ & $0.362 \pm 0.052$ & $58.33 \pm 5.46$ & 87.77 \\
\hline \multirow[t]{2}{*}{ Room temperature } & $\mathrm{F} 4$ & Day 1 & $237.56 \pm 33.9$ & $-24.0 \pm 4.49$ & $0.275 \pm 0.098$ & $58.80 \pm 6.34$ & 87.66 \\
\hline & & Day 30 & $236.66 \pm 34.9$ & $-24.5 \pm 3.92$ & $0.273 \pm 0.083$ & $58.21 \pm 5.48$ & 85.46 \\
\hline \multirow[t]{2}{*}{ Room temperature } & F7 & Day 1 & $231.34 \pm 37.8$ & $-28.9 \pm 5.92$ & $0.267 \pm 0.098$ & $58.73 \pm 6.24$ & 89.42 \\
\hline & & Day 30 & $232.44 \pm 239$ & $-28.7 \pm 4.95$ & $0.265 \pm 0.092$ & $57.34 \pm 6.64$ & 88.67 \\
\hline Refrigerator temperature & & Day 30 & $337.54 \pm 29.8$ & $-29.7 \pm 4.96$ & $0.261 \pm 0.082$ & $54.56 \pm 6.24$ & 87.54 \\
\hline \multirow[t]{2}{*}{ Refrigerator temperature } & $\mathrm{F} 4$ & Day 1 & $187.99 \pm 34.7$ & $-24.0 \pm 3.96$ & $0.275 \pm 0.097$ & $67.80 \pm 6.34$ & 87.66 \\
\hline & & Day 30 & $189.56 \pm 35.9$ & $-24.7 \pm 4.91$ & $0.273 \pm 0.056$ & $60.31 \pm 3.54$ & 86.39 \\
\hline \multirow[t]{2}{*}{ Refrigerator temperature } & F7 & Day 1 & $131.64 \pm 33.7$ & $-28.9 \pm 2.92$ & $0.267 \pm 0.089$ & $71.73 \pm 3.45$ & 89.42 \\
\hline & & Day 30 & $132.43 \pm 34.9$ & $-28.7 \pm 5.93$ & $0.266 \pm 0.062$ & $68.21 \pm 3.45$ & 88.48 \\
\hline
\end{tabular}

PDI: Polydispersity index

SLNs were examined for formulations F1, F4, and F7 by storing them at room temperatures $\left(40^{\circ} \mathrm{C} \pm 2^{\circ} \mathrm{C} / 75 \% \mathrm{RH} \pm 5 \%\right)$ and at refrigeration temperatures. The results of percentage drug EE, particle size, zeta potential, in vitro drug release, and physical appearance of the formulations were close to that of initial data with very slight variations suggesting that it has acceptable stability on storage.

\section{CONCLUSION}

In this study, a poorly water-soluble drug cilnidipine was successfully incorporated into SLNs by modified high shear homogenization and ultrasound techniques. The effects of different formulation variables on percentage EE and physiochemical properties were investigated. The in vitro release tests confirmed the prolongation of the drug release time showed the slow release of SLNs formulation. The type and concentration of surfactant, as well as the lipid matrix used, had a great influence on the physicochemical characterization of SLNs and the in vitro drug release. SLNs formulation F7 composed of Tween 80 as a surfactant and lower concentration of lipid matrix (50 mg of PA) showed the best result in view of the EE as well as in in vitro drug release. Particle size analysis showed that the formed particles were in nanosize and possessed a negative surface charge. Based on the observations it can be concluded that the formulated lipid nanoparticulate delivery system of cilnidipine could be widely accepted and physiologically safe lipids were capable of exhibiting sustained properties. Stability studies revealed that after 30 days of storage at different temperatures the mean diameters of SLNs remain practically the same which emphasizes the physical stability of these lipid particles. These data collectively support that SLNs are the promising delivery systems for poorly watersoluble drugs such as cilnidipine.

\section{AUTHOR'S CONTRIBUTIONS}

RPN carried out the formulation, in vitro characterization and drafted the manuscript. DN designed the protocol, participated and coordinated the study. All authors read and approved the final manuscript.

\section{ACKNOWLEDGMENT}

Authors are thankful to Madras Pharmaceuticals Pvt. Ltd., Chennai, India, for providing us with the gift sample of cilnidipine. Authors are also thankful to SRM Institute of Science and Technology, Kanchipuram, Tamil Nadu, India, for providing necessary infrastructure facilities required.

\section{CONFLICTS OF INTEREST}

Declared None

\section{REFERENCES}

1. Akter M, Banik S, Hossain MS. In vitro evaluation of oral extended release drug delivery system for metoprolol succinate using kollidon SR.
J App Pharma Sci 2012;25:188-92.

2. Kumar PS, Arivuchelvan A, Jagadeeswaran A, Subramanian N. Formulation, optimization and evaluation of enrofloxacin solid lipid nanoparticles for sustained oral delivery. Asian J Pharm Clin Res 2015;8:231-6

3. Christensen HR, Antonsen K, Simonsen K, Lindekaer A, Bonde J, Angelo HR, et al. Bioavailability and pharmacokinetics of isradipine after oral and intravenous administration: Half-life shorter than expected? Pharmacol Toxicol 2000;86:178-82.

4. Anton N, Benoit JP, Saulnier P. Design and production of nanoparticles formulated from nano-emulsion templates-a review. J Control Release 2008;128:185-99.

5. Radtke M, Muller RH. Nanostructured lipid drugcarriers. New Drugs 2001;2:48-52.

6. Jores K, Mehnert W, Bunjes H, Drechsler M, Mader K. Solid Lipid Nanoparticles (SLN) to Nanospoons. Visions and Reality of Colloidal Lipid Dispersions. Nuremberg, Germany: International Meeting on Pharmaceutics, Biopharmaceutics and Pharmaceutical Technology; 2008. p. $15-8$.

7. Saupe A, Gordon KV, Rades T. Structural investigations on nanoemulsions, solid lipid nanoparticles and nanostructured lipid carriers by cryofield emission scanning electron microscopy and raman spectroscopy. Int J Pharm 2006;314:56-62.

8. Venkateshwarlu V, Manjunath K. Preparation and in vitro release kinetics of clozapine solid lipid nanoparticles. J Controlled Release 2004;95:627-38.

9. Maruthi G, Smith AA, Manavalan R. Nanoparticles-a review. J Adv Sci Res 2011;2:12-29.

10. Monk JP, Campoli-Richards DM. Ofloxacin. A review of its antibacterial activity, pharmacokinetic properties and therapeutic use. Drugs 1987;33:346-91

11. Mishra R, Mir SR, Amin S. Polymeric nanoparticles for improved bioavailability of clinidipine. Int J Pharm Pharm Sci 2017;9:129-39.

12. Martinez M, McDermott P, Walker R. Pharmacology of the fluoroquinolones: A perspective for the use in domestic animals. Vet $\mathrm{J}$ 2006; 172:10-28

13. Ekambaram P, Sathali AA. Formulation and evaluation of solid lipid nanoparticles of ramipril. J Young Pharm 2011;3:216-20.

14. Saito A, Sawatari K, Fukuda Y, Nagasawa M, Koga H, Tomonaga A, et al. Susceptibility of legionella pneumophila to ofloxacin in vitro and in experimental legionella pneumonia in guinea pigs. Antimicrob Agents Chemother 1985;28:15-20.

15. Kumar BV, Babu BP, Prasad G. Formulation and evaluation of isradipine buccal tablets. Res J Pharm Tech 2012;5:1-10.

16. Todd PA, Ofloxacin FD. A reappraisal of its antimicrobial activity, pharmacology and therapeutic use. Drugs 1991;42:825-76.

17. Shelat P, Mandowara VK, Gupta DG, Patel S. Formulation of curcuminoid loaded solid lipid nanoparticles in order to improve oral bioavailability. Int J Pharm Pharm Sci 2015;7:278-2.

18. Priya S, Koland M, Kumara S. Formulation and characterization of ropinirole hydrochloride loaded solid lipid nanoparticles. Int J Pharm Pharm Sci 2015;7:85-9.

19. Prajapati ST, Maheshwari PD, Chhaganbhai N. Patel formulation and evaluation of orodispersible tablets of clinidipine by spray drying technique. World J of Pharm Pharm Sci 2015;45:1526-39.

20. Wang F, Gu XJ, Zhang MF, Tai TY. Treatment of typhoid fever with 
ofloxacin. J Antimicrobial Chemother 1989;23:785-8

21. Müller RH, Mäder K, Gohla S. Solid lipid nanoparticles (SLN) for controlled drug delivery-a review of the state of the art. Eur J Pharm Biopharm 2000;50:161-77.

22. Gamal A. Shazly ciprofloxacin controlled solid lipid nanoparticles characterization, in-vitro release and antibacterial activity assessment. Biomed Res Int 2017;2017:1-9.

23. Gaspar DP, Faria V, Gonçalves LM, Taboada P, Remuñán-López C, Almeida AJ, et al. Rifabutin-loaded solid lipid nanoparticles for inhaled antitubercular therapy: Physicochemical and in vitro studies. Int $\mathrm{J}$ Pharm 2016;497:199-209.

24. Gentry LO, Rodriguez-Gomez G. Ofloxacin versus parenteral therapy for chronic osteomyelitis. Antimicrob Agents Chemother 1991;35:538-41.

25. Sadiq AA, Rassol AA. A formulation and evaluation of silibinin loaded solid lipid nanoparticles for peroral use targeting lower part of gastrointestinal tract. Int J Pharm Pharm Sci 2014;1:55-67.

26. Jensen LB, Magnussson E, Gunnarsson L, Vermehren C, Nielsen HM, Petersson K. Corticosteroid solubility and lipid polarity control release from solid lipid nanoparticles. Int J Pharm 2010;390:53-60.

27. Schwarz C, Mehnert W. Solid lipid nanoparticles (SLN) for controlled drug delivery. II. Drug incorporation and physicochemical characterization. J Microencapsul 1999;16:205-13.
28. Zimmermann E, Müller RH, Mäder K. Influence of different parameters on reconstitution of lyophilized SLN. Int J Pharm 2000;196:211-3.

29. Havanoor SM. Isradipine loaded solid lipid nanoparticles for better treatment of hypertension - preparation, characterization and in vivo evaluation. International Journal of Biopharmaceutics 2014;5:218-24.

30. Okonogi S, Oguchi T, Yonemochi E, Puttipipatkhachorn S, Yamamoto K. Improved dissolution of ofloxacin via solid dispersion. Int J Pharm 1997;156:175-80.

31. Agro AS, Garner ET, Wright JW, de Escobar C, Villeda B, Seidlin M. Clinical trial of ototopical ofloxacin for treatment of chronic suppurative otitis media. Clin Ther 1998;20:744-59.

32. Al-Tahami K. Preparation, characterization, and in vitro release of ketoprofen loaded alginate microspheres. Int J Appl Pharm 2014;6:9-12.

33. Fogari R, Malacco E, Tettamanti F, Gnemmi AE, Milani M. Evening vs morning isradipine sustained release in essential hypertension: A double-blind study with $24 \mathrm{~h}$ ambulatory monitoring. Br J Clin Pharmacol 1993:35:51-4

34. Laxmi GA, Dachinamoorthy D, Babu YR, Surekha ML, Swamy GK. Development and validation of isradipine in bulk and its pharmaceutical formulation by the RP-HPLC method. Int Res J Pharm 2012;39:1-3.

35. Zhang L, Pornpattananangkul D, Hu CM, Huang CM. Development of nanoparticles for antimicrobial drug delivery. Curr Med Chem 2010;17:585-90. 